Hadron Structure '15

International Journal of Modern Physics: Conference Series

Vol. 39 (2015) 1560108 (20 pages)

(C) The Author(s)

DOI: $10.1142 /$ S2010194515601088

\title{
The renormalization scale problem and novel perspectives for QCD
}

\author{
Stanley J. Brodsky \\ SLAC National Accelerator Laboratory, Stanford University \\ Stanford, California 94039, USA \\ sjbth@slac.stanford.edu
}

Published 26 November 2015

\begin{abstract}
I discuss a number of novel tests of QCD, measurements which can illuminate fundamental features of hadron physics. These include the origin of the "ridge" in proton-proton collisions; the production of the Higgs at high $x_{F}$; the role of digluon-initiated processes for quarkonium production; flavor-dependent anti-shadowing; the effect of nuclear shadowing on QCD sum rules; direct production of hadrons at high transverse momentum; and leading-twist lensing corrections; and the breakdown of perturbative QCD factorization. I also review the "Principle of Maximum Conformalit" (PMC) which systematically sets the renormalization scale order-by-order in pQCD, independent of the choice of renormalization scheme, thus eliminating an unnecessary theoretical uncertainty.
\end{abstract}

\section{Introduction}

In the following sections I will discuss a number of physics topics which test novel aspects of QCD and, in some cases, confront conventional wisdom. These include:

- The origin of the "ridge" in proton-proton collisions

- Higgs production at high $x_{F}$

- Digluon-initiated quarkonium production

- Direct production of hadrons at high transverse momentum

- The possibility of flavor-dependent anti-shadowing

- Leading-twist lensing corrections and the breakdown of perturbative QCD factorization

- Nuclear shadowing and QCD sum rules

I will also review applications of the Principle of Maximum Conformality (PMC) which provides a systematic and unambiguous way to set the renormalization scale

This is an Open Access article published by World Scientific Publishing Company. It is distributed under the terms of the Creative Commons Attribution 3.0 (CC-BY) License. Further distribution of this work is permitted, provided the original work is properly cited. 
of any process at each order of PQCD. An unnecessary error from theory is eliminated. The PMC thus allows the colliders such as the LHC to test QCD much more precisely. The sensitivity of measurements to physics beyond the Standard Model is also greatly increased.

\section{The Principle of Maximum Conformality}

It has become conventional in perturbative QCD calculations to simply guess the renormalization scale $\mu$ of the QCD coupling $\alpha_{s}\left(\mu^{2}\right)$ and its range. This procedure is incorrect for QED, and it violates the principle of "renormalization group invariance": physical observables cannot depend on the choice of the renormalization scheme. Varying the renormalization scale can only expose terms in the pQCD series which are proportional to the $\beta$ function; varying the scale is thus an unreliable way to estimate the accuracy of pQCD predictions.

In fact, there is a rigorous method - the "Principle of Maximum Conformality" $(\mathrm{PMC})^{1,2}$ which systematically eliminates the renormalization scale uncertainty at each order of perturbation theory. The PMC provides scheme-independent predictions at each finite order in perturbative QCD by systematically identifying and shifting the nonconformal $\beta \neq 0$ terms into the QCD running coupling. The renormalization scale at each order of perturbation theory can thus be fixed by systematically identifying and resuming the nonconformal $\beta$ terms into the QCD running coupling.

In addition to scheme-independence (renormalization group invariance), the PMC satisfies these essential criteria:

- The Transitivity Property of the Renormalization Group: Predictions relating one observable to another cannot depend on the choice of the intermediate scheme.

- Commensurate Scale Relations: The PMC provides scale-fixed relations ${ }^{3}$ between observables such as the Generalized Crewther Relation ${ }^{4}$

- Distinct Scales and Number of Flavors at Each Order The argument of the running coupling and the number of effective quark flavors $n_{f}$ is fixed unambiguously and distinctly at each order of pQCD. This reflects the fact that the virtuality of the intermediate gluon and gluonic vertices is different at every order.

- Correct Conformal Limit: The predictions of conformal theory are reproduced when $\beta=0$.

- Multiple renormalization scales For example, the photon propagators in electronelectron elastic scattering at lowest order have virtuality $t$ and $u$.

- Elimination of the divergent renormalon series: The $\alpha_{s}^{n} \beta_{0}^{n} n \mid$ ! series associated with the $\beta$ function does not appear.

- Minimal dependence on the choice of the initial scale The dependence of PMC predictions on $\mu_{\text {init }}$ is found to be negligible.

- Correct Abelian limit: The PMC scale setting procedure reduces in the Abelian limit $^{5} N_{C} \rightarrow 0$ to the standard Gell-Mann-Low scale-setting procedure in QED. 
The PMC is a rigorous extension of the BLM method. ${ }^{6}$ The same principles that are used in QED also unambiguously determine the renormalization scale $\mu_{R}$ of the running coupling $\alpha_{s}\left(\mu_{R}^{2}\right)$ at each order of perturbation theory for QCD. The essential step: all $\beta$ terms in the pQCD series must be shifted into scales of the running couplings.

At low orders one can identify the $\beta$ terms from the occurrence of $n_{F}$ terms as proposed in the original BLM paper. ${ }^{6}$ at high orders one can use the $R_{\delta} \operatorname{method}^{7}$ : which systematically exposes all of the $\beta_{i}$ terms in any dimensional regularization scheme. One first generalizes the $\overline{M S}$ scheme by subtracting a constant $\delta$ in the dimensional regularization of the UV divergent amplitudes which renormalize the QCD running coupling, in addition to the usual $\log 4 \pi-\gamma_{E}$ subtraction. The resulting terms in $\delta$ reveal the pattern of nonconformal $\beta_{i} \neq 0$ terms. The renormalization scales are then fixed order-by-order by shifting the arguments of $\alpha_{s}^{n}$, so that no terms in $\delta$ or $\beta_{i}$ appear. The coefficients of the resulting series matches that of the corresponding scheme-independent $\beta=0$ conformal theory. The PMC predictions are thus independent of the choice of renormalization scheme, as required by renormalization group invariance. All other principles of the renormalization group, such as transitivity and reciprocity, are satisfied. ${ }^{3}$ The divergent "renormalon" $\alpha_{s}^{n} \beta_{0}^{n} n$ ! terms in the pQCD series based on a guessed scale are also eliminated.

The "Principle of Maximum Conformality" (PMC) $)^{2,8}$ gives predictions which rapidly converge and are independent of the choice of renormalization scheme; this is the key principle of renormalization group invariance. The PMC predictions are also independent of the choice of the initial scale $\mu_{R}$ to very high accuracy. As we have recently shown, ${ }^{9}$ the scale uncertainty for top-pair production at the LHC as a function of the $t \bar{t}$ invariant mass can be eliminated at the NNLO level. When one includes the known higher-order contributions, the central values predicted by the PMC become closer to the central value of the LHC measurements. The application of the PMC scale setting to Higgs branching ratios is given in Ref. 10 . In the case of the forward-backward asymmetry in $\bar{p} p \rightarrow t \bar{t} X$ at the Tevatron, the application of the $\mathrm{PMC}^{11}$ eliminates the anomaly reported by CDF and D0 - the discrepancy between measurements and pQCD predictions was based on the choice of an erroneous choice of renormalization scale and range. See Fig. 1. As in the QED analog, $e^{+} e^{-} \rightarrow \mu^{+} \mu^{-}$, the higher Born amplitudes which produce the $\mu^{+} \mu^{-}$ forward backward asymmetry have a smaller renormalization scale than the lowestorder amplitude. Thus it is essential to assign a different renormalization scale at each order of perturbation theory. The effective number of flavors $n_{f}$ is also different at each order.

There have been many successful applications of the BLM/PMC method. In the case of the forward-backward asymmetry in $\bar{p} p \rightarrow t \bar{t} X$ at the Tevatron, the application of the $\mathrm{PMC}^{11,12}$ eliminates the anomaly reported by $\mathrm{CDF}$ and $\mathrm{D} 0$ - the discrepancy between measurements and pQCD predictions was based on the choice of an erroneous choice of renormalization scale and range. As in its QED analog, $e^{+} e^{-} \rightarrow \mu^{+} \mu^{-}$, the higher Born amplitudes which produce the $\mu^{+} \mu^{-}$forward 


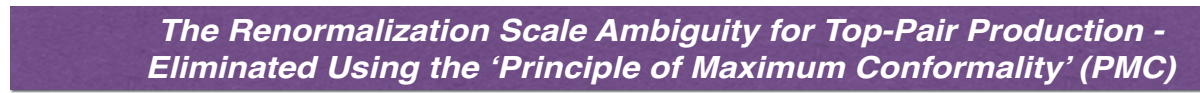

Eliminated Using the 'Principle of Maximum Conformality' (PMC)

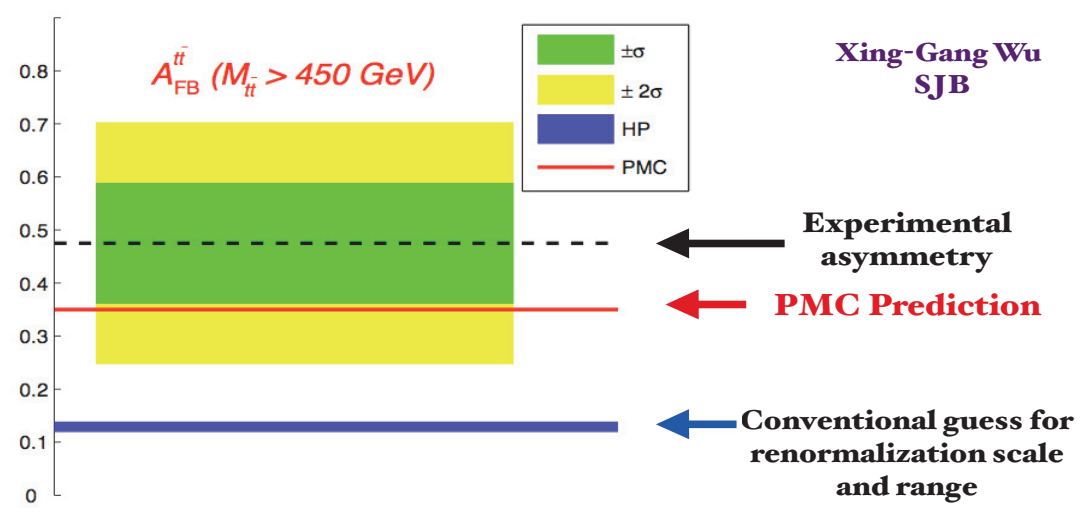

Top quark forward-backward asymmetry predicted by pQCD NNLO is within $1 \sigma$ of CDF/DO measurements using PMC/BLM scale setting

Fig. 1. Application of the PMC to $p \bar{p} \rightarrow t \bar{t} X$ forward-backward asymmetry.

backward asymmetry have a smaller renormalization scale than the lowest-order amplitude. Thus it is essential to assign a different renormalization scale at each order of perturbation theory. The effective number of flavors $n_{f}$ is also different at each order.

In summary: The purpose of the running coupling in any gauge theory is to sum all terms involving the $\beta$ function; in fact, when the renormalization scale $\mu$ is set properly, all non-conformal $\beta \neq 0$ terms in a perturbative expansion arising from renormalization are summed into the respective running coupling. The remaining terms in the perturbative series are then identical to that of a conformal theory; i.e., the theory with $\beta=0$. The divergent "renormalon" series of order $\alpha_{s}^{n} \beta^{n} n$ ! does not appear in the conformal series. Thus as in quantum electrodynamics, the renormalization scale $\mu$ is determined unambiguously by the "Principle of Maximal Conformality (PMC)". ${ }^{2,8}$ An important feature of the PMC is that its QCD predictions are independent of the choice of renormalization scheme. The PMC procedure also agrees with QED scale-setting in the Abelian $N_{C} \rightarrow 0$ limit. $^{5}$

The PMC provides a systematic and unambiguous way to set the renormalization scale of any process at each order of PQCD. An unnecessary error from theory is eliminated. The PMC thus allows the LHC to test QCD much more precisely, and the sensitivity of LHC measurements to physics beyond the Standard Model is greatly increased. The PMC is clearly an important advance for LHC physics since it provides an important opportunity to strengthen tests of fundamental theory. 


\section{The Origin of the "Ridge" in Proton-Proton Collisions and the Connection to the Dynamics Underlying Confinement}

The CMS collaboration ${ }^{13}$ at the LHC has reported a surprising phenomenon in very high-multiplicity high energy proton-proton collisions: a positive correlation between particles produced over a large rapidity interval along the same azimuthal angle as a trigger particle. It had been previously believed that such ridge-like correlations would only occur in nucleus-nucleus collisions due to their elliptical overlap. Bjorken, Goldhaber and $\mathrm{I}^{14}$ have suggested that the "ridge"-like correlation in $p p$ collisions reflects the rare events generated by the collision of aligned flux tubes which connect the valence quarks in the wave functions of the colliding protons. The "spray" of particles resulting from the approximate line source produced in such inelastic collisions then gives rise to events with a strong correlation between particles produced over a large range of both positive and negative rapidity. The highest multiplicity events will appears when the flux tubes have maximal overlap. In the case when the $Y$-junctions between the three quarks of each proton overlap, one could also generate a $v_{3}$ pattern.

The physics of colliding flux tubes can also be studied in high multiplicity, high energy electron-ion collisions at the proposed LHeC. In this case, the flux tube between the quark and antiquark of the virtual photon is oriented in azimuthal angle with the electron's scattering plane, and its characteristics - such as its size in impact space - can be controlled by the photon's virtuality, as well as the $q \bar{q}$ flavor.

The flux tube can be identified physically as the near-planar manifestation of the exchange of soft gluons which produce the fundamental color-confining interaction. In fact, the entire Regge spectrum of light-quark mesons and baryons in $n, L$, and $S$ is well reproduced by the eigenvalues of a frame-independent light-front Shrödinger and Dirac equations with a confining quark-diquark potential dictated by the softwall AdS/QCD approach and light-front holography. ${ }^{15-17}$ Remarkably the same confining light-front potential arises from principle of de Alfaro, Fubini, and Furlan ${ }^{18}$ which allows a mass scale an a confining harmonic oscillator potential to appear in equations of motion without affecting the conformal invariance of the action.

This description of hadron dynamics also predicts the shape of the light-front wavefunctions $\psi_{n, H}\left(x_{i}, k_{\perp i}\right)$ of the hadrons which underly hadronic form factors, structure functions, transverse momentum distributions, fragmentation functions, etc. In addition, diffractive vector meson electroproduction is predicted successfully without any new parameter. ${ }^{19}$

The use of Dirac's light-front time $\tau=t+z / c$ provides a Lorentz frameindependent formulation of hadronic collisions, where the quark and gluon composition of hadrons or their formation at the amplitude level is determined by their lightfront wavefunctions - the eigensolutions of the QCD light-front Hamiltonian. ${ }^{20} \mathrm{~A}$ remarkable feature of LFWFs is the fact that they are frame independent; i.e., the form of the LFWF is independent of the hadron's total momentum $P^{+}=P^{0}+P^{3}$ 
and $P_{\perp}$. The boost invariance of LFWFs contrasts dramatically with the complexity of boosting the wavefunctions defined at fixed time $t .{ }^{21}$ In this boost-invariant formalism, the light-front wavefunctions of the colliding protons (or ions) are frameindependent; there is no Lorentz contraction nor "colliding pancakes".

In the light-front framework, a hadron $H$ in QCD is identified as an eigenstate of the LF Hamiltonian $H_{L F}\left|\Psi_{H}\right\rangle=M_{H}^{2}\left|\Psi_{H}\right\rangle$, where $H_{L F}=P_{\mu} P^{\mu}=P^{-} P^{+}-P_{\perp}^{2}$ is the light-front time evolution operator which is derived directly from the Yang-Mills Lagrangian. The eigenvalues of this Heisenberg equation give the complete mass spectrum of the theory. The eigensolution $\left|\Psi_{H}\right\rangle$ projected on the free Fock basis provides the set of valence and non-valence light-front Fock state wavefunctions $\Psi_{n / H}\left(x_{i}, k_{\perp i}, \lambda_{i}\right)$, which describe the bound-state's internal momentum and spin distributions. If one quantizes the vector field in light-cone gauge $A^{+}=A^{0}+A^{3}=0$, the photons and gluons have physical polarization $S^{z}= \pm 1$; there are no ghosts, so that one has a physical interpretation of the bound states in terms of their fundamental constituents and spins. A new method, ${ }^{22}$ called "basis light-front quantization (BLFQ)", uses the orthonormal basis of eigensolutions of the AdS/QCD effective theory as the basis to diagonalize the full Hamiltonian of $\mathrm{QCD}(3+1)$. A comprehensive review is given in Ref. 23.

Hadron observables, such as hadronic structure functions, form factors, distribution amplitudes, GPDs, TMDs, and Wigner distributions can be computed as simple convolutions of light-front wavefunctions (LFWFs) in QCD. ${ }^{24}$ For example, one can calculate the electromagnetic and gravitational form factors $\left\langle p+q\left|j^{\mu}(0)\right| p\right\rangle$ and $\left\langle p+q\left|t^{\mu \nu}(0)\right| p\right\rangle$ of a hadron from the Drell-Yan-West formula - i.e., the overlap of LFWFs. The anomalous gravitomagnetic moment $B(0)$ defined from the spin-flip matrix element $\left\langle p+q\left|t^{\mu \nu}(0)\right| p\right\rangle$ at $q \rightarrow 0$ vanishes ${ }^{25}$ - consistent with the equivalence theorem of gravity. In contrast, in the instant form, the overlap of instant time wavefunctions is not sufficient. One must also couple the photon probe to currents arising spontaneously from the vacuum which are connected to the hadron's constituents.

The frame-independence of light-front quantization eliminates the complications from Lorentz boosts as well as vacuum processes. ${ }^{26,27} \mathrm{QCD}$ vacuum condensates do not appear. Implications for the cosmological constant are discussed in Ref. 26 .

\section{Higgs Production at High $x_{F}$ and the Intrinsic Heavy-Quark Distributions of the Proton}

A first-principle prediction of QCD is the existence of proton Fock states such as $|u u d Q \bar{Q}\rangle$. The light-wave function for such five-quark states have two origins: (1) the usual DGLAP "extrinsic" contribution arising from gluon splitting producing heavy quarks primarily at small momentum fractions $x=k^{+} / P^{+}$, and (2) the "intrinsic" contribution where the $Q \bar{Q}$ pair is multi-connected to the proton's valence quarks. ${ }^{28,29}$ The intrinsic contributions arise for example from the cut of the quark loop contribution to the $g g \rightarrow g g$ amplitude in the wavefunction 
describing proton's self energy. The amplitude for the intrinsic contribution is maximum at minimum off-shellness where the constituents tend have the same rapidity; i.e. $x_{i} \propto \sqrt{m_{i}^{2}+k_{\perp i}^{2}}$. Thus the intrinsic heavy quarks in the Fock state carry most of the hadron's momentum. Since the effective four-gluon operator is twist-six, the operator product expansion predicts the probability for the intrinsic five-quark state is proportional to $1 / m_{Q}^{2}$ in non-Abelian QCD. ${ }^{29,30}$ The intrinsic contribution also leads to asymmetries in the $s$ vs. $\bar{s}$ momentum and spin distributions. ${ }^{31}$ Recent reviews are given in Refs. 32 and 33.

The analog of intrinsic charm Fock states in hadrons ${ }^{28}$ such as $|u u d c \bar{c}\rangle$ in the proton is the $\left|e^{+} e^{-} \mu^{+} \mu^{-}\right\rangle$Fock state of positronium which appears through the cut of the muon-loop light-by-light contribution to the self energy of the positronium eigenstate. In this Fock state, the muons carry almost all of the momentum of the moving atom since the off-shell virtuality is minimal at equal velocity. In Abelian QED the probability for intrinsic lepton Fock states $\left|e^{+} e^{-} \ell \bar{\ell}\right\rangle$ in positronium scales as $1 / m_{\ell}^{4}$, whereas in QCD the probability of intrinsic heavy quarks in the wavefunction of a light hadron scales as $1 / m_{Q}^{2}$ because of its non-Abelian couplings. ${ }^{29,30}$

The conventional pQCD mechanisms for Higgs production at the LHC such as gluon fusion $g g \rightarrow H$ lead to Higgs boson production in the central rapidity region. However, the Higgs can also be produced at very high $x_{F}$ by the process $[Q \bar{Q}]+g \rightarrow H,{ }^{34}$ where both heavy quarks from the proton's five quark Fock state $|u u d Q \bar{Q}\rangle$ couple directly to the Higgs. See Fig. 2. Since the Higgs couples

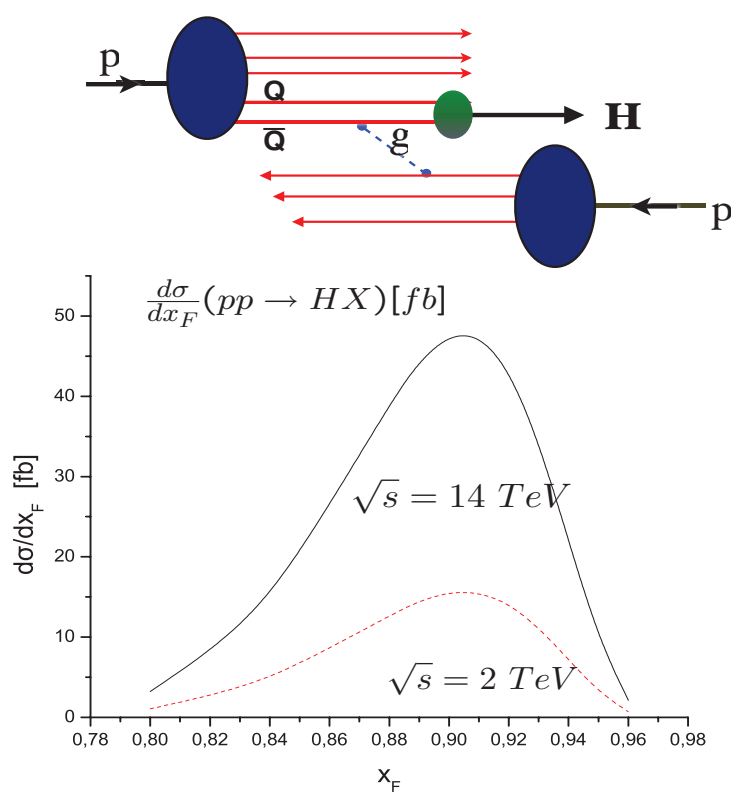

Fig. 2. Intrinsic Heavy Quark Mechanism and Cross section for Higgs Production at LHC and Tevatron energies. 
to each quark proportional to its mass, one has roughly equal contributions from intrinsic $s \bar{s}, c \bar{c}, b \bar{b}$ and even $t \bar{t}$ Fock states. The intrinsic heavy-quark distribution of the proton at high $x$ leads to Higgs production with as much as $80 \%$ of the beam momentum. The same mechanism produces the $J / \psi$ at high $x_{F}$ as observed in fixed-target experiments such as NA3. The decay of the high- $x_{F}$ decay Higgs to muons could be observed using very forward detectors at the LHC. The predicted cross section $d \sigma / d x_{F}(p p \rightarrow H X)$ for Higgs production at high $x_{F} \sim 0.8$ computed by Kopeliovitch, Schmidt, Goldhaber, and myself ${ }^{34}$ is of order of $50 \mathrm{fb}$. We have also computed with Soffer ${ }^{35}$ the corresponding double-diffractive rate $p p \rightarrow H p p X$. Testing these predictions would open up a new domain of Higgs physics at the LHC.

Signals for the intrinsic heavy quark distribution at high $x$ have been seen by many experiments, including the measurement of $c\left(x, Q^{2}\right)$ at high $x$ by the EMC collaboration and forward production at high $x_{F}$ of the $\Lambda_{c}$ at the ISR and SELEX at FermiLab, $\Lambda_{b}$, at the ISR, double-charm baryons by SELEX, and the high $x_{F}$ production of the $J / \psi$ and double $J / \psi$ by NA3 at CERN at FermiLab. A review and references are given in Ref. 36. Since the $[Q \bar{Q}]$ is a color-octet $8_{C}$, the production of the $J / \psi$ at high $x_{F}$ from intrinsic charm in $p A$ collisions occurs at the nuclear front surface, explaining the observed $A^{2 / 3}$ nuclear dependence. Intrinsic charm in the reaction $g c \rightarrow \gamma c$ has the potential to explain the anomalously large rate seen by D0 $p \bar{p} \rightarrow c \gamma X$ at high $p_{T}^{\gamma}$. Intrinsic charm is included in CTEQ 6.6M. A signal for "intrinsic strangeness" $s(x)+\bar{s}(x)$ in the region $0.1<x<0.4$ from HERMES has been discussed by Chang and Peng. ${ }^{37}$ The duality of the $|u u d s \bar{s}\rangle$ Fock state with hadronic channels such as $|K \Lambda\rangle$ implies that the $s(x)$ and $\bar{s}(x)$ distributions will be different in shape and spin content. ${ }^{31}$

\section{Digluon-Initiated Quarkonium Production}

Since the $J / \psi$-nucleon cross section is only a few millibarns, the usual expectation is that the $J / \psi$ production cross sections in nuclei will be approximately linear in the number of nucleons $A$. However, the production cross section $p A \rightarrow J / \psi X$ measured by $\mathrm{LHCb}^{38}$ and $\mathrm{ALICE}^{39}$ at forward rapidity $y \sim 4$ shows unexpectedly strong nuclear suppression. This strong suppression cannot be accounted for by shadowing of the nuclear gluon distribution.

Arleo and Peigne ${ }^{40,41}$ have suggested that the strong nuclear suppression of $J / \psi$ production in $p A$ collisions can be explained as a manifestation of the "color-octet" model: the $c \bar{c}$ propagates through the nucleus as a color-octet, and its energy loss will be proportional to its energy if the induced gluon radiation is coherent on the entire nucleus. The color-octet $c \bar{c}$ is assumed to convert to the color-singlet $J / \psi$ after exciting the nucleus.

There is an alternative QCD mechanism for producing the $J / \psi$ in proton-nucleus collisions at forward rapidity and small transverse momentum; digluon-initiated subprocesses: $[g g] g \rightarrow J / \psi$. Here the $[g g]$ is a color-octet digluon originating from the colliding proton; e.g. from its $|u u d g g\rangle$ Fock state. See Fig. 3. It should be noted 


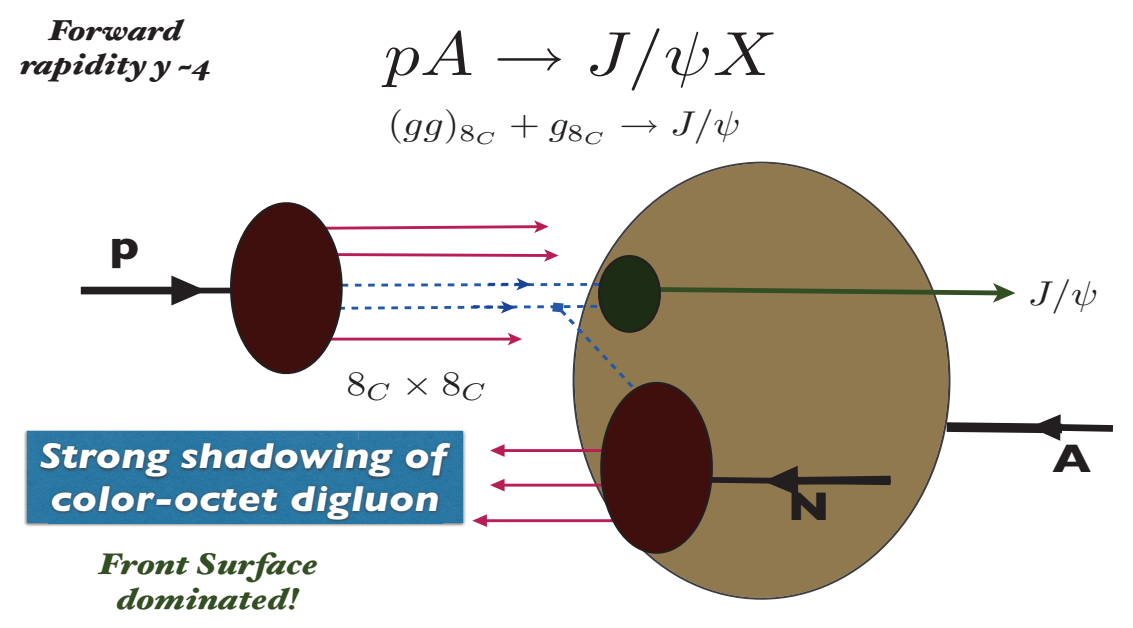

\section{Crossing: Diffractive \& pomeron exchange}

Digluon-initiated subprocess!

Fig. 3. Digluon-induced quarkonium production. Since the color-octet digluon is strongly shadowed, the production process occurs dominantly on the front surface of the nuclear target.

that since $g\left(x, Q^{2}\right)$ falls rapidly, two gluons in the digluon, each with $x \sim 0.02$, could have a higher probability than a single gluon with $x \sim 0.04$. The digluon mechanism will be suppressed at high $p_{T}$, but can dominate at low $p_{T}$ and forward rapidity. The propagating color-octet digluon has a large interaction cross section, and it thus interacts primarily at the nucleus front surface, giving a production cross section $\sigma(p A \rightarrow J / \psi X) \propto A^{2 / 3}$. The produced $J / \psi$ then propagates essentially freely though the nuclear interior. The $\Upsilon$ produced by di-gluons will have a similar $A$ dependence. However, this is not the case for the $\psi^{\prime}$; it can be further suppressed as it propagates through the nuclear environment because of its larger radius. This digluon subprocess is the color-octet analog of the color-singlet two-gluon exchange mechanism $^{42}$ underlying diffractive processes such as $\ell p \rightarrow \ell p X$. The digluon multiparton subprocess is also analogous to the higher-twist subprocess $[q] q q \rightarrow \gamma^{*} q$ which dominates the $\pi N \rightarrow \ell \bar{\ell} X$ Drell-Yan reaction at high $x_{F}$ and is known to accounts for the observed dramatic change from transverse to longitudinal virtual photon polarization. ${ }^{43}$ Similarly, multiparton "direct" subprocesses can account ${ }^{44}$ for the observed anomalous power-law fall-off of high $p_{T}$ inclusive hadron production cross sections $E d \sigma /{ }^{3} p(p p \rightarrow h X)$ at fixed $x_{T}=2 p_{T} / \sqrt{s}$ and fixed $\theta_{C M}$ as discussed in the next section.

The $p A \rightarrow J / \psi X$ cross sections measured in fixed-target experiments at CERN and FermiLab at high $x_{F}$ also show a very strong nuclear suppression at high $x_{F}$. The ratio of the nuclear and proton target cross sections has the form $A^{\alpha\left(x_{F}\right)}$, where $x_{F}$ is Feynman fractional longitudinal momentum of the $J / \psi$. At small $x_{F}, \alpha\left(x_{F}\right)$ is slightly smaller than one, but at $x_{F} \sim 1$, it decreases to $\alpha=2 / 3$. These results are 
again surprising since (1) the dependence $A^{\alpha}=A^{2 / 3}$ is characteristic of a strongly interacting hadron, not a small-size quarkonium state; and (2) the functional dependence $A^{\alpha\left(x_{F}\right)}$ contradicts pQCD factorization. ${ }^{45}$ As noted in the previous section, the observed nuclear suppression, in combination with the anomalously nearly flat cross section at high $x_{F}$ can be explained by the properties of the intrinsic charm Fock state. ${ }^{34,36}$ QCD predicts that the color-configuration of the heavy quark pair $Q \bar{Q}$ in the intrinsic five-quark Fock state is primarily a color-octet. The intrinsic heavy quark Fock state of the proton: $\left|(u u d)_{8_{C}}(c \bar{c})_{8_{C}}\right\rangle$ thus interacts primarily with the $A^{2 / 3}$ nucleons at the front surface because of the large color-dipole moment of the color-octet $c \bar{c}$. The color-singlet quarkonium state is thus produced at the front surface, and it then propagates through the nucleus with high $x_{F}$.

\section{The Role of Direct Processes in the Production of Hadrons at High $p_{T}$}

The factorization picture derived from pQCD has played a guiding role in virtually all aspects of hadron physics phenomenology. In the case of inclusive reactions such as $p p \rightarrow \pi X$, the pQCD ansatz predicts that the cross section at leading order in pion's transverse momentum $p_{T}$ can be computed by convoluting the perturbatively calculable hard subprocess cross section with the process-independent structure functions and quark fragmentation functions. It is thus usually assumed that hadrons produced at high transverse momentum in inclusive high energy hadronic collisions arise dominantly from jet fragmentation. A fundamental test of leading-twist QCD predictions in high transverse momentum hadronic reactions is the measurement of the power-law fall-off of the inclusive cross section ${ }^{46}$ $E d \sigma / d^{3} p(A B \rightarrow C X)=F\left(\theta_{c m}, x_{T}\right) / p_{T}^{n_{\text {eff }}}$ at fixed $x_{T}=2 p_{T} / \sqrt{s}$ and fixed $\theta_{C M}$. In the case of the scale-invariant parton model $n_{\mathrm{eff}}=4$. However in QCD, $n_{\mathrm{eff}} \sim 4+\delta$ where $\delta \simeq 1.5$ is the typical correction to the conformal prediction arising from the QCD running coupling and the DGLAP evolution of the input parton distribution and fragmentation functions. ${ }^{44,47}$ The usual expectation then is that leadingtwist subprocesses (i.e., the leading power-law contributions) will dominate high $p_{T}$ hadron production at RHIC and at Tevatron energies. Measurements of isolated photon production $p p \rightarrow \gamma_{\text {direct }} X$, as well as jet production, do agree well with the leading-twist scaling prediction $n_{\mathrm{eff}} \simeq 4.5 .{ }^{44}$ However, measurements of $n_{\text {eff }}$ for hadron production show much faster fall-off in $p_{T}$ at fixed $x_{T}$ and $\theta_{C M}$ and are inconsistent with the leading twist predictions. Striking deviations from the leading-twist predictions were also observed at lower energy at the ISR and Fermilab fixed-target experiments. ${ }^{46,48,49}$ This deviation points to a significant contribution from 'direct' higher-twist processes where the hadron is created directly in the hard subprocess, rather than from quark or gluon jet fragmentation.

Normally many more pions than protons are produced at high transverse momentum in hadron-hadron collisions. This is also true for the peripheral collisions of heavy ions. However, when the nuclei collide with maximal overlap (central 
collisions) the situation is reversed - more protons than pions emerge. This observation at $\mathrm{RHIC}^{50}$ contradicts the usual expectation that protons should be more strongly absorbed than pions in the nuclear medium.

In fact, a significant fraction of high $p_{\perp}^{H}$ isolated hadrons can emerge directly from hard higher-twist subprocess ${ }^{44,47}$ even at the LHC. The direct production of hadrons can also explain ${ }^{51}$ the remarkable "baryon anomaly" observed at RHIC: the ratio of baryons to mesons at high $p_{\perp}^{H}$, as well as the power-law fall-off $1 / p_{\perp}^{n}$ at fixed $x_{\perp}=2 p_{\perp} / \sqrt{s}$, both increase with centrality, ${ }^{50}$ opposite to the usual expectation that protons should suffer more energy loss in the nuclear medium than mesons. The high values $n_{\text {eff }}$ with $x_{T}$ seen in the data indicate the presence of an array of higher-twist processes, including subprocesses where the hadron enters directly, rather than through jet fragmentation. ${ }^{52}$ Although they are suppressed by powers of $1 / p_{T}$, the direct higher twist processes can dominate because they are energy efficient — no same-side energy or momentum is lost from the undetected fragments. Thus the incident colliding partons are evaluated at the minimum possible values of light-front momentum fractions $x_{1}$ and $x_{2}$, where the parton distribution functions are numerically large. Since these processes create color-transparent baryons with minimal absorption, this mechanism can explain the RHIC baryon anomaly. ${ }^{51}$

\section{Flavor-Dependent Anti-Shadowing}

It has been conventional to assume that the nuclear modifications to the structure functions measured in deep inelastic charged lepton-nucleus and neutrino-nucleus interactions are identical. The antishadowing of the nuclear structure functions is particularly interesting. Empirically, one finds $F_{2 A}\left(x, Q^{2}\right) /(A / 2) F_{d}\left(x, Q^{2}\right)>1$ in the domain $0.1<x<0.2$; i.e., the measured nuclear structure function (referenced to the deuteron) is larger than the scattering on a set of $A$ independent nucleons.

One can show ${ }^{53}$ using Gribov-Glauber theory that the Bjorken-scaling diffractive deep inelastic scattering events lead to the shadowing of nuclear structure func-

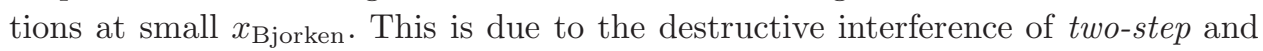
one-step amplitudes in the nucleus. Since diffraction involves rescattering, one sees that shadowing and diffractive processes are not intrinsic properties of hadron and nuclear wavefunctions and structure functions, but are properties of the complete dynamics of the scattering reaction. ${ }^{54}$ In fact, Gribov-Glauber theory also predicts that the antishadowing of nuclear structure functions is not universal, but depends on the quantum numbers of each struck quark and antiquark. ${ }^{55}$ This could explain the recent observation of Schienbein et $a l .{ }^{56}$ who find that the nuclear structure functions in the range $0.1<x<0.2$ measured by $\mathrm{NuTeV}$ in deep inelastic neutrino charged-current reactions differ significantly from the distributions measured in deep inelastic electron and muon scattering.

Note that there are leading-twist diffractive contributions $\gamma^{*} N_{1} \rightarrow(q \bar{q}) N_{1}$ arising from Reggeon exchanges in the $t$-channel. For example, isospin-non-singlet 
$C=+$ Reggeons contribute to the difference of proton and neutron structure functions, giving the characteristic Kuti-Weiskopf $F_{2 p}-F_{2 n} \sim x^{1-\alpha_{R}(0)} \sim x^{0.5}$ behavior at small $x$. The $x$ dependence of the structure functions reflects the Regge behavior $\nu^{\alpha_{R}(0)}$ of the virtual Compton amplitude at fixed $Q^{2}$ and $t=0$. The phase of the diffractive amplitude is determined by analyticity and crossing to be proportional to $-1+i$ for $\alpha_{R}=0.5$, which together with the phase from the Glauber cut, leads to constructive interference of the diffractive and nondiffractive multi-step nuclear amplitudes. The coherence length only needs to be long enough to ensure coherence between the one-step and two-step Glauber processes, not the entire nuclear length. The nuclear structure function is predicted ${ }^{57}$ to be enhanced precisely in the domain $0.1<x<0.2$ where antishadowing is empirically observed.

Since quarks of different flavors couple to different Reggeons, this leads to the remarkable prediction that nuclear antishadowing is not universal ${ }^{55}$ instead, it depends on the quantum numbers of the struck quark. This picture implies substantially different antishadowing for charged and neutral current reactions as indicated by $\mathrm{NuTeV}$ measurements. See Fig. 4.

\section{Leading-Twist Lensing Corrections and the Breakdown of pQCD Factorization Rules}

The effects of the final-state interactions of the scattered quark in deep inelastic scattering have been traditionally assumed to either give an inconsequential phase

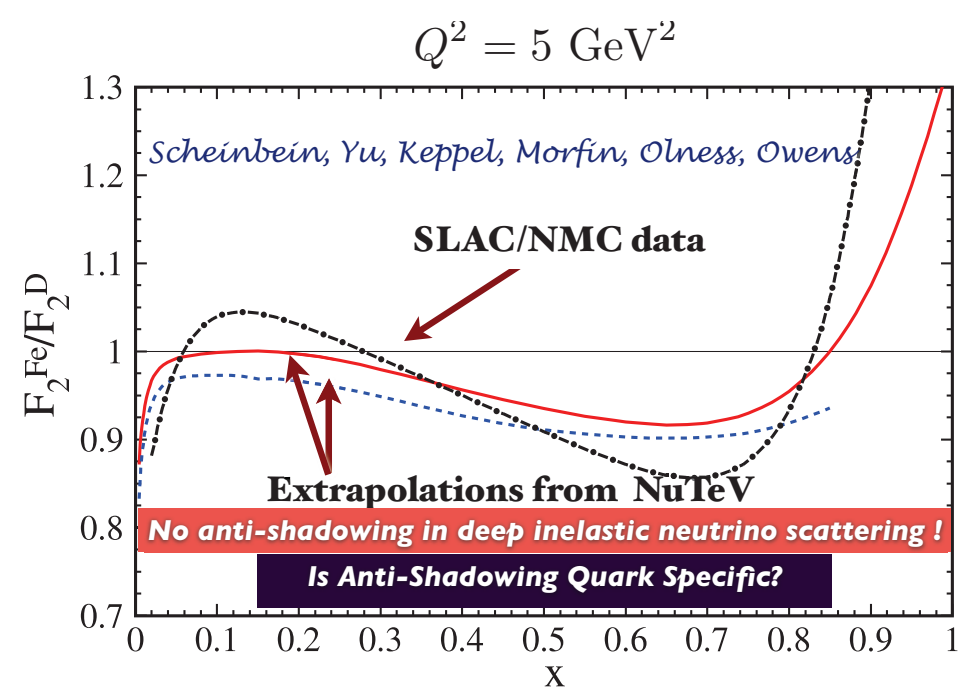

Fig. 4. Comparison of nuclear structure functions measured in charge and neutral current deep inelastic lepton scattering. The $\mathrm{NuTeV}$ charged current measurement $\mu A \rightarrow \nu X$ scattering does not appear to show antishadowing. The compilation is from Schienbein, et al. ${ }^{58}$ 
factor or power-law suppressed corrections. However, this is only true for sufficiently inclusive cross sections. For example, consider semi-inclusive deep inelastic lepton scattering (SIDIS) on a polarized target $\ell p_{\uparrow} \rightarrow H \ell^{\prime} X$. In this case the final-state gluonic interactions of the scattered quark lead to a $T$-odd non-zero spin correlation of the plane of the lepton-quark scattering plane with the polarization of the target proton $^{59}$ which is not power-law suppressed with increasing virtuality of the photon $Q^{2}$; i.e. it Bjorken-scales. This leading-twist "Sivers effect" 60 is nonuniversal in the sense that pQCD predicts an opposite-sign correlation in Drell-Yan reactions relative to single-inclusive deep inelastic scattering. ${ }^{61,62}$ This important but yet untested prediction occurs because the Sivers effect in the Drell-Yan reaction is modified by the initial-state interactions of the annihilating antiquark. A simple argument for this sign change is given in Ref. 63 .

Similarly, the final-state interactions of the produced quark with its comoving spectators in SIDIS produces a final-state $T$-odd polarization correlation - the "Collins effect". This can be measured without beam polarization by measuring the correlation of the polarization of a hadron such as the $\Lambda$ baryon with the quarkjet production plane. Analogous spin effects occur in QED reactions due to the rescattering via final-state Coulomb interactions. Although the Coulomb phase for a given partial wave is infinite, the interference of Coulomb phases arising from different partial waves leads to observable effects. These considerations have led to a reappraisal of the range of validity of the standard factorization ansatz. ${ }^{64}$

The calculation of the Sivers single-spin asymmetry in deep inelastic lepton scattering in QCD requires two different orbital angular momentum components: $S$ wave with the quark-spin parallel to the proton spin and $P$-wave for the quark with anti-parallel spin; the difference between the final-state "Coulomb" phases leads to a $\vec{S} \cdot \vec{q} \times \vec{p}$ correlation of the proton's spin with the virtual photon-to-quark production plane. ${ }^{59}$ Thus, as it is clear from its QED analog, the final-state gluonic interactions of the scattered quark lead to a $T$-odd non-zero spin correlation of the plane of the lepton-quark scattering plane with the polarization of the target proton. ${ }^{59}$

The $S$ - and $P$-wave proton wavefunctions also appear in the calculation of the Pauli form factor quark-by-quark. Thus one can correlate the Sivers asymmetry for each struck quark with the anomalous magnetic moment of the proton carried by that quark, ${ }^{65}$ leading to the prediction that the Sivers effect is larger for positive pions as seen by the HERMES experiment at DESY, ${ }^{66}$ the COMPASS experiment $^{67-69}$ at CERN, and CLAS at Jefferson Laboratory ${ }^{70,71}$ The physics of the "lensing dynamics" or Wilson-line physics ${ }^{72}$ underlying the Sivers effect involves nonperturbative quark-quark interactions at small momentum transfer, not the hard scale $Q^{2}$ of the virtuality of the photon. It would interesting to see if the strength of the soft initial- or final-state scattering can be predicted using the effective confining potential of QCD from light-front holographic QCD.

Measurements $^{73}$ of the Drell-Yan Process $\pi p \rightarrow \mu^{+} \mu^{-} X$ display an angular distribution which contradicts pQCD expectations. In particular one observes an anomalously large $\cos 2 \phi$ azimuthal angular correlation between the lepton decay 
plane and its production plane which contradicts the Lam-Tung relation, a prediction of perturbative QCD factorization. ${ }^{74}$ Such effects again point to the importance of initial and final-state interactions of the hard-scattering constituents, ${ }^{75}$ corrections not included in the standard pQCD factorization formalism. For example, if both the quark and antiquark in the Drell-Yan subprocess $q \bar{q} \rightarrow \mu^{+} \mu^{-}$interact with the spectators of the other hadron, then one predicts a $\cos 2 \phi \sin ^{2} \theta$ planar correlation in unpolarized Drell-Yan reactions. ${ }^{75}$ This "double Boer-Mulders effect" can account for the anomalously large $\cos 2 \phi$ correlation observed by the NA10 collaboration $^{73}$ and the violation ${ }^{75,76}$ of the Lam Tung relation a standard prediction based on perturbative QCD factorization. ${ }^{74}$ Such effects point to the importance of both initial and final-state interactions of the hard-scattering constituents, corrections not included in the standard pQCD factorization formalism. One also observes large single-spin asymmetries in reactions such as $p p_{\uparrow} \pi X$, an effect not yet explained. ${ }^{77}$ Another important signal for factorization breakdown at the LHC will be the observation of a $\cos 2 \phi$ planar correlation in dijet production. As emphasized by Collins and Qiu, ${ }^{64}$ the traditional factorization formalism of perturbative QCD fails in detail for many hard inclusive reactions because of initial- and final-state interactions.

The final-state interactions of the struck quark with the spectators ${ }^{42}$ also lead to diffractive events in deep inelastic scattering (DDIS) at leading twist, such as $\ell p \rightarrow \ell^{\prime} p^{\prime} X$, where the proton remains intact and isolated in rapidity; in fact, approximately $10 \%$ of the deep inelastic lepton-proton scattering events observed at HERA are diffractive. ${ }^{78,79}$ This seems surprising since the underlying hard subprocess $\ell q \rightarrow \ell^{\prime} q^{\prime}$ is highly disruptive of the target nucleon. The presence of a rapidity gap between the target and diffractive system requires that the target remnant emerges in a color-singlet state; this is made possible in any gauge by the soft rescattering incorporated in the Wilson line or by augmented light-front wavefunctions. Quite different fractions of single $p p \rightarrow$ Jet $p^{\prime} X$ and double diffractive $p \bar{p} \rightarrow$ Jet $p^{\prime} \bar{p}^{\prime} X$ events are observed at the Tevatron. The underlying mechanism is believed to be soft gluon exchange between the scattered quark and the remnant system in the final state occurring after the hard scattering occurs.

\section{Is the Momentum Sum Rule Valid for Nuclear Structure Functions?}

Sum rules for DIS processes are analyzed using the operator product expansion of the forward virtual Compton amplitude, assuming it reduces in the limit $Q^{2} \rightarrow \infty$ to matrix elements of local operators such as the energy-momentum tensor. The moments of the structure function and other distributions can then be evaluated as overlaps of the target hadron's light-front wavefunction (LFWF), the hadronic eigensolution of the LF Hamiltonian, as in the Drell-Yan-West formulae for hadronic form factors. ${ }^{80-83}$ The phases of the resulting DIS amplitude and OPE matrix elements reflect the real phase of the stable target hadron's wavefunction. This 
approximation defines the "static" contribution" 54,84 to the measured parton distribution functions (PDF), transverse momentum distributions, etc. The resulting momentum, spin and other sum rules reflect the properties of the hadron's lightfront wavefunction.

However, as discussed in the previous section, final-state interactions which occur after the lepton-quark scattering, give non-trivial contributions to deep inelastic scattering processes at leading twist and survive at high $Q^{2}$ and high $W^{2}=(q+p)^{2}$. For example, the pseudo- $T$-odd Sivers effect ${ }^{59}$ is directly sensitive to the rescattering of the struck quark. Similarly, diffractive deep inelastic scattering involves the exchange of a gluon after the quark has been struck by the lepton. ${ }^{42}$ These "lensing" corrections survive when both $W^{2}$ and $Q^{2}$ are large since the vector gluon couplings grow with energy. Part of the phase can be associated with a Wilson line as an augmented $\mathrm{LFWF}^{72}$ which do not affect the moments.

The Glauber propagation of the vector system $V$ produced by the diffractive DIS interaction on the front face and its inelastic interaction with the nucleons in the nuclear interior $V+N_{b} \rightarrow X$ occurs after the lepton interacts with the struck quark. Because of the rescattering dynamics, the DDIS amplitude acquires a complex phase from Pomeron and Regge exchange; thus final-state rescattering corrections lead to nontrivial "dynamica" contributions to the measured PDFs; i.e., they involve physics aspects of the scattering process itself. ${ }^{63}$

Diffractive DIS is leading-twist and is the essential component of the two-step amplitude which causes shadowing and antishadowing of the nuclear PDF. It is important to analyze whether the momentum and other sum rules derived from the OPE expansion in terms of local operators remain valid when these dynamical rescattering corrections to the nuclear PDF are included. The OPE is derived assuming that the LF time separation between the virtual photons in the forward virtual Compton amplitude $\gamma^{*} A \rightarrow \gamma^{*} A$ scales as $1 / Q^{2}$. However, the propagation of the vector system $V$ produced by the diffractive DIS interaction on the front face and its inelastic interaction with the nucleons in the nuclear interior $V+N_{b} \rightarrow X$ are characterized by a longer LF time which scales as $1 / W^{2}$. Thus the leading-twist multi-nucleon processes that produce shadowing and antishadowing in a nucleus are evidently not present in the $Q^{2} \rightarrow \infty$ OPE analysis.

It should be emphasized that shadowing in deep inelastic lepton scattering on a nucleus involves the nucleons at or near the front surface - i.e., the nucleons facing the incoming lepton beam. This geometrical bias is not built into the frame-independent nuclear LFWFs used to evaluate the matrix elements of local currents. Thus the dynamical phenomena of leading-twist shadowing and antishadowing appear to invalidate the sum rules for nuclear PDFs. The same complications occur in the leading-twist analysis of deeply virtual Compton scattering $\gamma^{*} A \rightarrow \gamma^{*} A$ on a nuclear target. The observed difference of the nuclear distribution measured charged current versus neutral current deep inelastic scattering on nuclei shown in Fig. 4 also challenges the validity of OPE sum rules for nuclear targets. 


\section{S. J. Brodsky}

\section{Acknowledgments}

Presented by SJB at Hadron Structure' 15, Horny Smokovec, Slovak Republic, June 26 to July 3, 2015. I thank my collaborators for many contributions to this report. I also thank Paul Hoyer, Simonetta Liuti, and Adam Szczepaniak for helpful discussions on the effect of shadowing on nuclear distributions. This research was supported by the Department of Energy, contract DE-AC02-7c6SF00515. SLACPUB-16403.

\section{References}

1. S. J. Brodsky, M. Mojaza and X. G. Wu, "Systematic Scale-Setting to All Orders: The Principle of Maximum Conformality and Commensurate Scale Relations," Phys. Rev. D 89, 1, 014027 (2014) [arXiv:1304.4631 [hep-ph]].

2. S. J. Brodsky and L. Di Giustino, "Setting the Renormalization Scale in QCD: The Principle of Maximum Conformality," Phys. Rev. D 86, 085026 (2012) [arXiv:1107.0338 [hep-ph]].

3. S. J. Brodsky and H. J. Lu, "Commensurate scale relations in quantum chromodynamics," Phys. Rev. D 51, 3652 (1995) [hep-ph/9405218].

4. S. J. Brodsky, G. T. Gabadadze, A. L. Kataev and H. J. Lu, "The Generalized Crewther relation in QCD and its experimental consequences," Phys. Lett. B 372, 133 (1996) [hep-ph/9512367].

5. S. J. Brodsky and P. Huet, "Aspects of $\mathrm{SU}(\mathrm{N}(\mathrm{c}))$ gauge theories in the limit of small number of colors," Phys. Lett. B 417, 145 (1998) [hep-ph/9707543].

6. S. J. Brodsky, G. P. Lepage and P. B. Mackenzie, "On the Elimination of Scale Ambiguities in Perturbative Quantum Chromodynamics," Phys. Rev. D 28, 228 (1983).

7. M. Mojaza, S. J. Brodsky and X. G. Wu, "Systematic All-Orders Method to Eliminate Renormalization-Scale and Scheme Ambiguities in Perturbative QCD," Phys. Rev. Lett. 110, 192001 (2013) [arXiv:1212.0049 [hep-ph]].

8. S. J. Brodsky and X. G. Wu, "Scale Setting Using the Extended Renormalization Group and the Principle of Maximum Conformality: the QCD Coupling Constant at Four Loops," Phys. Rev. D 85, 034038 (2012) [Phys. Rev. D 86, 079903 (2012)] [arXiv:1111.6175 [hep-ph]].

9. S. J. Brodsky and X. G. Wu, "Application of the Principle of Maximum Conformality to Top-Pair Production," Phys. Rev. D 86, 014021 (2012) [Phys. Rev. D 87, 9, 099902 (2013)] [arXiv:1204.1405 [hep-ph]].

10. S. Q. Wang, X. G. Wu, X. C. Zheng, J. M. Shen and Q. L. Zhang, "The Higgs boson inclusive decay channels $H \rightarrow b \bar{b}$ and $H \rightarrow g g$ up to four-loop level," Eur. Phys. J. $C$ 74, 4, 2825 (2014) [arXiv:1308.6364 [hep-ph]].

11. S. J. Brodsky and X. G. Wu, "Application of the Principle of Maximum Conformality to the Top-Quark Forward-Backward Asymmetry at the Tevatron," Phys. Rev. D 85, 114040 (2012) [arXiv:1205.1232 [hep-ph]].

12. S. Q. Wang, X. G. Wu, Z. G. Si and S. J. Brodsky, "Application of the Principle of Maximum Conformality to the Top-Quark Charge Asymmetry at the LHC," Phys. Rev. D 90, 11, 114034 (2014) [arXiv:1410.1607 [hep-ph]].

13. V. Khachatryan et al. [CMS Collaboration], "Observation of Long-Range Near-Side Angular Correlations in Proton-Proton Collisions at the LHC," JHEP 1009, 091 (2010) [arXiv:1009.4122 [hep-ex]]. 
14. J. D. Bjorken, S. J. Brodsky and A. Scharff Goldhaber, "Possible multiparticle ridgelike correlations in very high multiplicity proton-proton collisions," Phys. Lett. B 726, 344 (2013) [arXiv:1308.1435 [hep-ph]].

15. S. J. Brodsky, G. F. de Teramond, H. G. Dosch and J. Erlich, "Light-Front Holographic QCD and Emerging Confinement," Phys. Rept. 584, 1 (2015) [arXiv:1407.8131 [hep$\mathrm{ph}]]$.

16. S. J. Brodsky, G. F. de Teramond and H. G. Dosch, "Threefold Complementary Approach to Holographic QCD," Phys. Lett. B 729, 3 (2014) [arXiv:1302.4105 [hepth]].

17. S. J. Brodsky and G. F. de Teramond, "Hadronic spectra and light-front wavefunctions in holographic QCD," Phys. Rev. Lett. 96, 201601 (2006) [hep-ph/0602252].

18. V. de Alfaro, S. Fubini and G. Furlan, "Conformal Invariance in Quantum Mechanics," Nuovo Cim. A 34, 569 (1976).

19. J. R. Forshaw and R. Sandapen, "An AdS/QCD holographic wavefunction for the rho meson and diffractive rho meson electroproduction," Phys. Rev. Lett. 109, 081601 (2012) [arXiv:1203.6088 [hep-ph]].

20. B. L. G. Bakker et al., "Light-Front Quantum Chromodynamics: A framework for the analysis of hadron physics," Nucl. Phys. Proc. Suppl. 251-252, 165 (2014) [arXiv:1309.6333 [hep-ph]].

21. S. J. Brodsky and J. R. Primack, "The Electromagnetic Interactions of Composite Systems," Annals Phys. 52, 315 (1969).

22. J. P. Vary, X. Zhao, A. Ilderton, H. Honkanen, P. Maris and S. J. Brodsky, "Basis light-front quantization: A new approach to non-perturbative scattering and timedependent production processes," Acta Phys. Polon. Supp. 6, 257 (2013).

23. S. J. Brodsky, H. C. Pauli and S. S. Pinsky, "Quantum chromodynamics and other field theories on the light cone," Phys. Rept. 301, 299 (1998) [hep-ph/9705477].

24. B. Pasquini and C. Lorc, "Wigner Distributions in Light-Front Quark Models," Few Body Syst. 55, 287 (2014).

25. S. J. Brodsky, D. S. Hwang, B. Q. Ma and I. Schmidt, "Light cone representation of the spin and orbital angular momentum of relativistic composite systems," Nucl. Phys. B 593, 311 (2001) [hep-th/0003082].

26. S. J. Brodsky and R. Shrock, "Standard-Model Condensates and the Cosmological Constant," arXiv:0803.2554 [hep-th].

27. S. J. Brodsky, C. D. Roberts, R. Shrock and P. C. Tandy, "Confinement contains condensates," Phys. Rev. C 85, 065202 (2012) [arXiv:1202.2376 [nucl-th]].

28. S. J. Brodsky, P. Hoyer, C. Peterson and N. Sakai, "The Intrinsic Charm of the Proton," Phys. Lett. B 93, 451 (1980).

29. S. J. Brodsky, J. C. Collins, S. D. Ellis, J. F. Gunion and A. H. Mueller, "Intrinsic Chevrolets At The SSC," DOE/ER/40048-21 P4, SLAC-PUB-15471.

30. M. Franz, M. V. Polyakov and K. Goeke, "Heavy quark mass expansion and intrinsic charm in light hadrons," Phys. Rev. D 62, 074024 (2000) [hep-ph/0002240].

31. S. J. Brodsky and B. Q. Ma, "The Quark / anti-quark asymmetry of the nucleon sea," Phys. Lett. B 381, 317 (1996) [hep-ph/9604393].

32. S. J. Brodsky and S. Gardner, "Comment on "New Limits on Intrinsic Charm in the Nucleon from Global Analysis of Parton Distributions"," arXiv:1504.00969 [hep-ph].

33. S. J. Brodsky, A. Kusina, F. Lyonnet, I. Schienbein, H. Spiesberger and R. Vogt, "A review of the intrinsic heavy quark content of the nucleon," arXiv:1504.06287 [hep-ph].

34. S. J. Brodsky, A. S. Goldhaber, B. Z. Kopeliovich and I. Schmidt, "Higgs Hadroproduction at Large Feynman x," Nucl. Phys. B 807, 334 (2009) [arXiv:0707.4658 [hep$\mathrm{ph}]]$. 
35. S. J. Brodsky, B. Kopeliovich, I. Schmidt and J. Soffer, "Diffractive Higgs production from intrinsic heavy flavors in the proton," Phys. Rev. D 73, 113005 (2006) [hep$\mathrm{ph} / 0603238]$.

36. S. J. Brodsky, "Novel Heavy Quark Phenomena in QCD," PoS Hadron 2013, 013 (2013) [arXiv:1401.5886 [hep-ph]].

37. W. C. Chang and J. C. Peng, "Extraction of Various Five-Quark Components of the Nucleons," Phys. Lett. B 704, 197 (2011) [arXiv:1105.2381 [hep-ph]].

38. R. Aaij et al. [LHCb Collaboration], "Study of $J / \psi$ production and cold nuclear matter effects in $p P b$ collisions at $\sqrt{s_{N N}}=5 \mathrm{TeV}$," JHEP 1402, 072 (2014) [arXiv:1308.6729 [nucl-ex]].

39. K. Aamodt et al. [ALICE Collaboration], "Suppression of Charged Particle Production at Large Transverse Momentum in Central Pb-Pb Collisions at $\sqrt{s_{N N}}=2.76 \mathrm{TeV}$," Phys. Lett. B 696, 30 (2011) [arXiv:1012.1004 [nucl-ex]].

40. F. Arleo and S. Peigne, "J/ $\psi$ suppression in $\mathrm{p}-\mathrm{A}$ collisions from parton energy loss in cold QCD matter," Phys. Rev. Lett. 109, 122301 (2012) [arXiv:1204.4609 [hep-ph]].

41. F. Arleo and S. Peigne, "Heavy-quarkonium suppression in p-A collisions from parton energy loss in cold QCD matter," JHEP 1303, 122 (2013) [arXiv:1212.0434 [hep-ph]].

42. S. J. Brodsky, P. Hoyer, N. Marchal, S. Peigne and F. Sannino, "Structure functions are not parton probabilities," Phys. Rev. D 65, 114025 (2002) [hep-ph/0104291].

43. E. L. Berger and S. J. Brodsky, "Quark Structure Functions of Mesons and the DrellYan Process," Phys. Rev. Lett. 42, 940 (1979).

44. F. Arleo, S. J. Brodsky, D. S. Hwang and A. M. Sickles, "Higher-Twist Dynamics in Large Transverse Momentum Hadron Production," Phys. Rev. Lett. 105, 062002 (2010) [arXiv:0911.4604 [hep-ph]].

45. P. Hoyer, M. Vanttinen and U. Sukhatme, "Violation of factorization in charm hadroproduction," Phys. Lett. B 246, 217 (1990).

46. D. W. Sivers, S. J. Brodsky and R. Blankenbecler, "Large Transverse Momentum Processes," Phys. Rept. 23, 1 (1976).

47. F. Arleo, S. J. Brodsky, D. S. Hwang and A. M. Sickles, "Higher-twist contributions to large $p_{\perp}$ hadron production in hadronic collisions," arXiv:1006.4045 [hep-ph].

48. J. W. Cronin, H. J. Frisch, M. J. Shochet, J. P. Boymond, P. A. Piroue and R. L. Sumner, "Production of Hadrons with Large Transverse Momentum at 200-GeV and 300GeV.," Phys. Rev. Lett. 31, 1426 (1973).

49. D. Antreasyan, J. W. Cronin, H. J. Frisch, M. J. Shochet, L. Kluberg, P. A. Piroue and R. L. Sumner, "Production of Hadrons at Large Transverse Momentum in 200-GeV, 300-GeV and 400-GeV p p and p n Collisions," Phys. Rev. D 19, 764 (1979).

50. S. S. Adler et al. [PHENIX Collaboration], "Scaling properties of proton and antiproton production in $\mathrm{s}(\mathrm{NN})^{* *}(1 / 2) 200-\mathrm{GeV} \mathrm{Au}+\mathrm{Au}$ collisions," Phys. Rev. Lett. 91, 172301 (2003) [nucl-ex/0305036].

51. S. J. Brodsky and A. Sickles, "The Baryon Anomaly: Evidence for Color Transparency and Direct Hadron Production at RHIC," Phys. Lett. B 668, 111 (2008) [arXiv:0804.4608 [hep-ph]].

52. R. Blankenbecler, S. J. Brodsky and J. F. Gunion, "Analysis of Particle Production at Large Transverse Momentum," Phys. Rev. D 12, 3469 (1975).

53. L. Stodolsky, "Rapidity gap' events and shadowing in deep inelastic scattering," Phys. Lett. B 325, 505 (1994).

54. S. J. Brodsky, "Dynamic versus Static Structure Functions and Novel Diffractive Effects in QCD," AIP Conf. Proc. 1105, 315 (2009) [arXiv:0811.0875 [hep-ph]].

55. S. J. Brodsky, I. Schmidt and J. J. Yang, "Nuclear antishadowing in neutrino deep inelastic scattering," Phys. Rev. D 70, 116003 (2004) [hep-ph/0409279]. 
56. I. Schienbein, J. Y. Yu, C. Keppel, J. G. Morfin, F. I. Olness and J. F. Owens, "Parton distribution function uncertainties and nuclear corrections for the LHC," arXiv:0806.0723 [hep-ph].

57. S. J. Brodsky and H. J. Lu, "Shadowing and Antishadowing of Nuclear Structure Functions," Phys. Rev. Lett. 64, 1342 (1990).

58. I. Schienbein, J. Y. Yu, C. Keppel, J. G. Morfin, F. Olness and J. F. Owens, "Nuclear parton distribution functions from neutrino deep inelastic scattering," Phys. Rev. D 77, 054013 (2008) [arXiv:0710.4897 [hep-ph]].

59. S. J. Brodsky, D. S. Hwang and I. Schmidt, "Final state interactions and single spin asymmetries in semiinclusive deep inelastic scattering," Phys. Lett. B 530, 99 (2002) [hep-ph/0201296].

60. D. W. Sivers, "Single Spin Production Asymmetries from the Hard Scattering of Point-Like Constituents," Phys. Rev. D 41, 83 (1990).

61. J. C. Collins, "Leading twist single transverse-spin asymmetries: Drell-Yan and deep inelastic scattering," Phys. Lett. B 536, 43 (2002) [hep-ph/0204004].

62. S. J. Brodsky, D. S. Hwang and I. Schmidt, "Initial state interactions and single spin asymmetries in Drell-Yan processes," Nucl. Phys. B 642, 344 (2002) [hep-ph/0206259].

63. S. J. Brodsky, D. S. Hwang, Y. V. Kovchegov, I. Schmidt and M. D. Sievert, "SingleSpin Asymmetries in Semi-inclusive Deep Inelastic Scattering and Drell-Yan Processes," Phys. Rev. D 88, 1, 014032 (2013) [arXiv:1304.5237 [hep-ph]].

64. J. Collins and J. W. Qiu, " $k_{T}$ factorization is violated in production of high-transversemomentum particles in hadron-hadron collisions," Phys. Rev. D 75, 114014 (2007) [arXiv:0705.2141 [hep-ph]].

65. Z. Lu and I. Schmidt, "Connection between the Sivers function and the anomalous magnetic moment," Phys. Rev. D 75, 073008 (2007) [hep-ph/0611158].

66. A. Airapetian et al. [HERMES Collaboration], "Single-spin asymmetries in semiinclusive deep-inelastic scattering on a transversely polarized hydrogen target," Phys. Rev. Lett. 94, 012002 (2005) [hep-ex/0408013].

67. F. Bradamante [COMPASS Collaboration], "New COMPASS results on Collins and Sivers asymmetries," Nuovo Cim. C 035N2, 107 (2012) [arXiv:1111.0869 [hep-ex]].

68. M. G. Alekseev et al. [COMPASS Collaboration], "Measurement of the Collins and Sivers asymmetries on transversely polarised protons," Phys. Lett. B 692, 240 (2010) [arXiv:1005.5609 [hep-ex]].

69. F. Bradamante, "Transverse spin and transverse momentum effects at COMPASS," Mod. Phys. Lett. A 24, 3015 (2009).

70. H. Avakian et al. [CLAS Collaboration], "Measurement of Single and Double Spin Asymmetries in Deep Inelastic Pion Electroproduction with a Longitudinally Polarized Target," Phys. Rev. Lett. 105, 262002 (2010) [arXiv:1003.4549 [hep-ex]].

71. H. Gao et al., "Transverse Spin Structure of the Nucleon through Target Single Spin Asymmetry in Semi-Inclusive Deep-Inelastic $\left(e, e^{\prime} \pi^{ \pm}\right)$Reaction at Jefferson Lab," Eur. Phys. J. Plus 126, 2 (2011) [arXiv:1009.3803 [hep-ph]].

72. S. J. Brodsky, B. Pasquini, B. W. Xiao and F. Yuan, "Phases of Augmented Hadronic Light-Front Wave Functions," Phys. Lett. B 687, 327 (2010) [arXiv:1001.1163 [hep$\mathrm{ph}]$.

73. S. Falciano et al. [NA10 Collaboration], "Angular Distributions of Muon Pairs Produced by 194-GeV/c Negative Pions," Z. Phys. C 31, 513 (1986).

74. C. S. Lam and W. K. Tung, "A Parton Model Relation Sans QCD Modifications in Lepton Pair Productions," Phys. Rev. D 21, 2712 (1980).

75. D. Boer, S. J. Brodsky and D. S. Hwang, "Initial state interactions in the unpolarized Drell-Yan process," Phys. Rev. D 67, 054003 (2003) [hep-ph/0211110]. 
76. D. Boer, "Investigating the origins of transverse spin asymmetries at RHIC," Phys. Rev. D 60, 014012 (1999) [hep-ph/9902255].

77. Z. T. Liang and T. C. Meng, "Meson production mechanisms and single spin hadron hadron collisions," Phys. Rev. D 49, 3759 (1994).

78. C. Adloff et al. [H1 Collaboration], "Inclusive measurement of diffractive deep inelastic ep scattering," Z. Phys. C 76, 613 (1997) [hep-ex/9708016].

79. J. Breitweg et al. [ZEUS Collaboration], "Measurement of the diffractive cross-section in deep inelastic scattering using ZEUS 1994 data," Eur. Phys. J. C 6, 43 (1999) [hep-ex/9807010].

80. S. J. Brodsky and S. D. Drell, "The Anomalous Magnetic Moment and Limits on Fermion Substructure," Phys. Rev. D 22, 2236 (1980).

81. S. Liuti, A. Rajan, A. Courtoy, G. R. Goldstein and J. O. Gonzalez Hernandez, "Partonic Picture of GTMDs," Int. J. Mod. Phys. Conf. Ser. 25, 1460009 (2014) [arXiv:1309.7029 [hep-ph]].

82. C. Mondal and D. Chakrabarti, "Generalized parton distributions and transverse densities in a light-front quarkdiquark model for the nucleons," Eur. Phys. J. C 75, no. 6, 261 (2015) [arXiv:1501.05489 [hep-ph]].

83. C. Lorce, B. Pasquini and M. Vanderhaeghen, "Unified framework for generalized and transverse-momentum dependent parton distributions within a 3Q light-cone picture of the nucleon," JHEP 1105, 041 (2011) [arXiv:1102.4704 [hep-ph]].

84. S. J. Brodsky, "Dynamic versus Static Hadronic Structure Functions," Nucl. Phys. A 827, 327C (2009) [arXiv:0901.0781 [hep-ph]].

85. G. Grunberg, "Renormalization Scheme Independent QCD and QED: The Method of Effective Charges," Phys. Rev. D 29, 2315 (1984).

86. M. Gell-Mann and F. E. Low, "Quantum electrodynamics at small distances," Phys. Rev. 95, 1300 (1954).

87. S. J. Brodsky, M. S. Gill, M. Melles and J. Rathsman, "An Analytic extension of the MS-bar renormalization scheme," Phys. Rev. D 58, 116006 (1998) [hep-ph/9801330]. 